\title{
Vessel Centerline Tracking in CTA and MRA Images Using Hough Transform
}

\author{
Maysa M.G. Macedo ${ }^{1}$, Choukri Mekkaoui ${ }^{2}$, and Marcel P. Jackowski ${ }^{1}$ \\ ${ }^{1}$ University of São Paulo, Department of Computer Science, \\ Rua do Matão 1010, São Paulo, Brazil \\ \{maysa, mjack\}@ime.usp.br \\ ${ }^{2}$ Harvard Medical School, 25, Shattuck Street, Boston, MA, USA \\ mekkaoui@nmr.mgh. harvard.edu
}

\begin{abstract}
Vascular disease is characterized by any condition that affects the circulatory system. Recently, a demand for sophisticated software tools that can characterize the integrity and functional state of vascular networks from different vascular imaging modalities has appeared. Such tools face significant challenges such as: large datasets, similarity in intensity distributions of other organs and structures, and the presence of complex vessel geometry and branching patterns. Towards that goal, this paper presents a new approach to automatically track vascular networks from CTA and MRA images. Our methodology is based on the Hough transform to dynamically estimate the centerline and vessel diameter along the vessel trajectory. Furthermore, the vessel architecture and orientation is determined by the analysis of the Hessian matrix of the CTA or MRA intensity distribution. Results are shown using both synthetic vessel datasets and real human CTA and MRA images. The tracking algorithm yielded high reproducibility rates, robustness to different noise levels, associated with simplicity of execution, which demonstrates the feasibility of our approach.
\end{abstract}

Keywords: lumen segmentation, vessel tracking, Hough transform, angiographic images, CTA, MRA.

\section{Introduction}

Vascular diseases have reached a significant number of people in the world. Developed countries and more recently underdeveloped countries have shown an increase of aneurysms, stenosis, embolisms and arteritis [1. In this context, successful detection and characterization of vessel anomalies by medical imaging modalities as Computed Tomography Angiography (CTA) and Magnetic Resonance Angiography (MRA), requires efficient computational tools for visualization and analysis. Vessel segmentation methods may provide useful surgical planning information and may aid in diagnostics [2]3. There are important challenges involving the characterization of vascular architectures: highresolution images that increases computational time and storage space; handling

I. Bloch and R.M. Cesar, Jr. (Eds.): CIARP 2010, LNCS 6419, pp. 295-302, 2010.

(C) Springer-Verlag Berlin Heidelberg 2010 
of bifurcations and successful detection of capillaries. According to [4] the analysis of vascular images can be divided into four steps : (i) feature extraction - detect vessel points and diameters; (ii) geometric model - connect the vessel points to form vascular trees; (iii) quantify properties of the vascular tree; and in the case of serial imaging; and (iv) quantify differences in these properties over time. In most cases, methods rely on the detection of local tubular structures based on the local intensity characteristics. The feature extraction process in 3D images can be performed by tracking the vessel centerlines [56] or by extracting the vessel wall [7]. While most centerline-based methods directly estimate vessel diameters, they may have limitations such as their inability to track more than one branch at a time. Model-based methods, on the other hand, estimate vessel diameter from boundary detection techniques and may suffer from discontinuities because of noise. They may also require non-trivial initialization procedures. Overviews on vessel lumen segmentation techniques are presented in [8] and [9], according to different categories.

In order to avoid common drawbacks of model-based methods, we propose a methodology that easily tracks vessel centerlines and estimate vessel diameters, has simple initialization and avoids cumbersome multiscale extraction techniques. The work presented in this paper focuses on the development of a technique to extract blood vessel morphological attributes from 3D medical images using Hessian matrix information coupled with Hough Transform (HT) in order to perform detection and tracking.

This paper proposes the use of the HT to determine vessel scale for successful tracking without resorting to time-consuming multiscale techniques. Successive circle detection on $2 \mathrm{D}$ cross-sections and prediction of vessel direction are performed in order to track along a branch. In the next section, the methodology is detailed, followed by synthetic and real datasets results. A discussion of the methodology followed by conclusions and future research directions are provided at the end.

\section{Vessel Tracking Method}

The goal of the proposed vessel tracking is to construct a vessel skeleton based on the extracted centerlines on several slices. CTA and MRA images show vessels as high intensity profiles, with maximum intensity near their centers. The idea is to model this maximum intensity location as the center of a circle. When the plane of extraction is completely orthogonal to the vessel, the vessel wall can be modeled as a circle and this process can be repeated by following the tangent of the vessel trajectory.

\subsection{Initialization and Preprocessing}

An extraction plane orthogonal to a given vessel is selected from a $3 \mathrm{D}$ image to start the tracking process. Following selection, the extracted plane is smoothed by an anisotropic diffusion filter 1011 to reduce noise and enhance linear 
structures. Next, morphological opening and subtraction operators are applied to highlight maximum intensities and the Canny filter finally is employed in order to extract edges. Parameter values at each step of this pipeline is chosen depending on the modality.

\subsection{Vessel Detection by Hough Transform}

Once the edge image is obtained, the HT is computed to identify circle centers and their estimated diameters. If more than one vessel is found in the chosen plane of extraction, the best match is chosen. At end of this step, both centerline and vessel radius is acquired.

\subsection{Vessel Tangent Estimation}

Based on this centerline and on the detected radius taken as a vessel scale, eigenvalues $\left(\lambda_{1}, \lambda_{2}, \lambda_{3}\right)$ and eigenvectors $\left(\boldsymbol{e}_{1}, \boldsymbol{e}_{2}, \boldsymbol{e}_{3}\right)$ are computed from a Hessian matrix computed at the centerline location. The eigenvector corresponding to the eigenvalue closer to $0\left(\lambda_{1}\right)$ indicates the vessel direction $\left(\boldsymbol{e}_{1}\right)$.This direction is maintained by the multiplication of $\boldsymbol{e}_{1}$ and the sign of the dot product of $\boldsymbol{e}_{1}$ and the previous tangent direction $\boldsymbol{t}_{i-1}$ at centerline point $x_{i}$ :

$$
\boldsymbol{t}_{i}=\operatorname{signal}\left(\boldsymbol{e}_{1} \cdot \boldsymbol{t}_{i-1}\right) \boldsymbol{e}_{1}
$$

The direction $\boldsymbol{t}_{i}$ defines the normal to the next extraction plane in the 3D image. Image resampling according to the new direction is necessary to detect the circles in a perpendicular manner. This process finishes when it is not possible to detect a significant difference between the eigenvalues. The proposed tracking method consists of successive executions of the above steps to extract a vessel trajectory. Figure 1 depicts tracking process scheme. Initially, only one vessel will be detected for each $2 \mathrm{D}$ cross-section to start the tracking. The idea is detect all centerlines of a unique vessel branch and then go back to each branching point to continue the centerline detection.

According to Aylward [12] it is necessary recover from local discontinuities, so during the tracking we adopted the following heuristics:

1) If $\lambda_{2}$ and $\lambda_{3}$ of the local Hessian become negative, the tangent and the normal directions may temporarily change. We detect the next eigenvector that best matches the previous tangent direction modifying the equation (11) to (3).

2) If $\left|\lambda_{1}-\lambda_{2}\right|<0.02$ the tangent vessel direction is not well defined. It is necessary choose the eigenvector that best matches the previous tangent direction according to equation (3).

$$
\begin{gathered}
q=\arg \max _{j \in 1 \ldots 3}\left(\left|\boldsymbol{e}_{j} \cdot \boldsymbol{t}_{i-1}\right|\right) \\
\boldsymbol{t}_{i}=\operatorname{signal}\left(\boldsymbol{e}_{q} \cdot \boldsymbol{t}_{i-1}\right) \boldsymbol{e}_{q}
\end{gathered}
$$




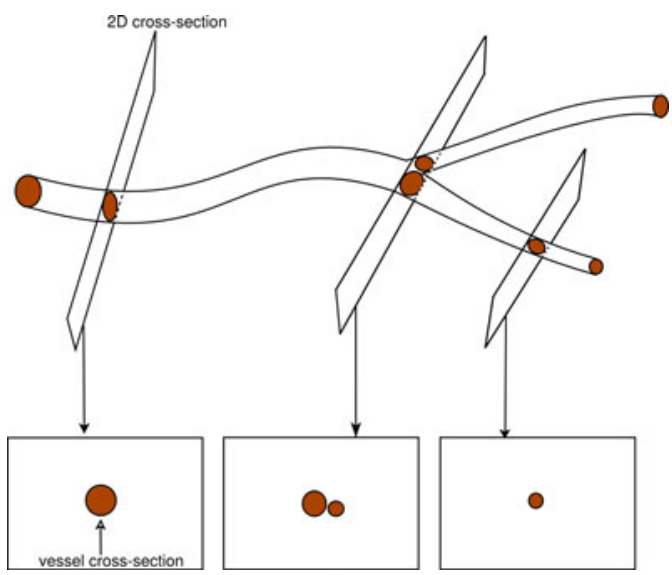

Fig. 1. Representative scheme of proposed method. The arrows indicate extracted planes according to the tangent direction identified by Hessian matrix.

\subsection{Bifurcations}

In a vessel network, a bifurcation is the branching of a vessel, which it can be further divided into several branches. This feature constitutes a challenge for most vessel tracking algorithm because the eigenvalues are not able to represent each individual bifurcation. However, when the tracking trajectory approaches a branching point, the vessel profile starts to change in shape, as well as its curvature (Fig. 2). The vessel shape changes from a convex configuration and becomes concave. This changing is employed to identify branching locations and determine follow-up directions for the subsequent extraction planes.

\section{Preliminary Results}

The methodology was implemented using $\mathrm{C}++$ language and auxiliary libraries such as Visualization Toolkit and Insight Toolkit. Both synthetic images and a real CTA and MRA image was used in order to assess whether the centerlines and vessel tangent directions are correctly extracted.

\subsection{Synthetic Images}

Synthetic vessel images were constructed and corrupted by Gaussian noise in order to test for method's robustness to noise. In addition, to assess the tracking method's behavior on different vessel shapes, these images depict shapes with low and high curvature. Noise quantity depended on a normal random number and a percentage related to the maximum intensity image. In this case, images with $5 \%, 10 \%$ and $15 \%$ of noise were used. Synthetic images have size $128 \times 128$ $\times 128$, containing vessel of radius 2 voxels wide and cubic resolution $\left(1 \mathrm{~mm}^{3}\right)$. For 

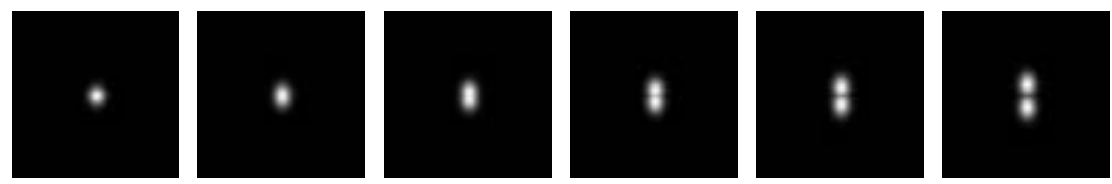

Fig. 2. Tracking sequence of a synthetic vessel with a branching point. From left to right, this sequence shows each slice when the tracking is approaching the bifurcation. A simple circle (convex curve) becomes a non trivial form (concave curve).

(a)

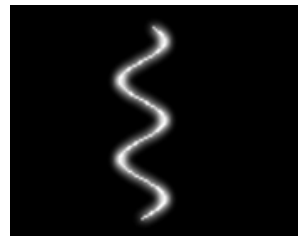

(c)

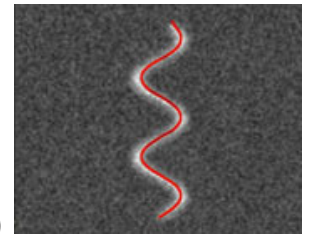

(b)

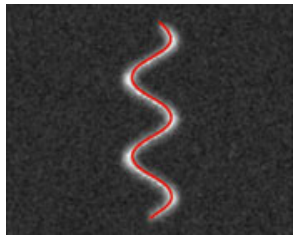

(d)

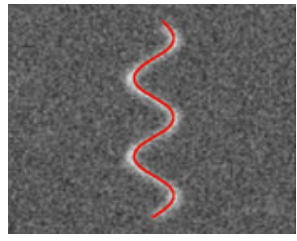

Fig. 3. Centerlines extracted from a simulated sinusoid. (a) Original image. (b) Image with $5 \%$ of noise. (c) Image with $10 \%$ of noise. (d) Image with $15 \%$ of noise.

our set of sinusoidal synthetic images, the average distance to the ground-truth centerlines was $1.5 \mathrm{~mm}$. Figures 3 and 4 show the extracted tube trajectories from noisy images.

\subsection{Real Images}

The real images represent a thoracic CTA and a cerebral MRA. Authors were blind in regard of the presence or not of possible vessel anomalies in either dataset. The CTA image was released by Centre Hospitalier Universitaire Hopital de la Timone from a GE LightSpeed Pro 16 with size $512 \times 512 \times 256$, resolution $\left(0.78 \times 0.78 \times 0.78 \mathrm{~mm}^{3}\right)$. The MRA image was acquired by Institute of Radiology - Hospital das Clínicas - University of São Paulo in a Philips 3T with size $512 \times 512 \times 290$ and resolution $\left(0.39 \times 0.39 \times 0.50 \mathrm{~mm}^{3}\right)$. The goal is to extract the thoracic aorta from the CTA dataset and a segment of the internal carotid from the MRA image.

Preprocessing steps are ilustrated by Figure 5(a)-(e). Following preprocessing, the HT is performed and the result is displayed by Figure 5 (f). After identifying the vessel center, the direction of the vessel cross-section is determined by computing the Hessian matrix. Figure 6 shows a sequence of resampled images according to the vessel direction characterizing the vessel tracking approach. Note that the aorta is at the middle of the image. As the $2 \mathrm{D}$ cross-section is 
(a)

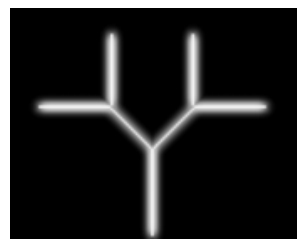

(c)

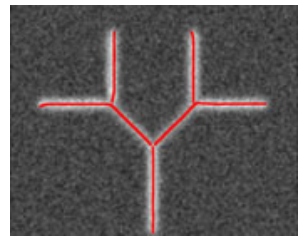

(b)

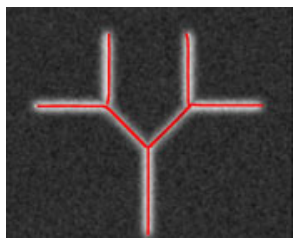

(d)

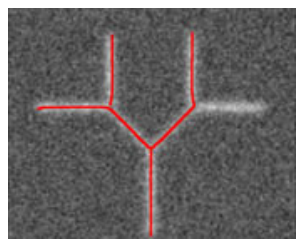

Fig. 4. Centerlines extracted from synthetic image with bifurcation points. (a) Original image. (b) Image with $5 \%$ of noise. (c) Image with $10 \%$ of noise. (d) Image with $15 \%$ of noise. In this case one of branches was not identified.

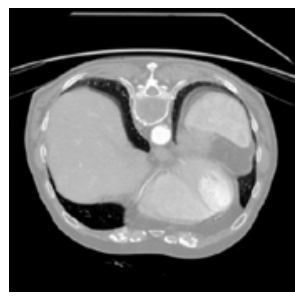

(a)

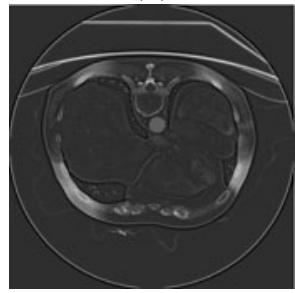

(d)

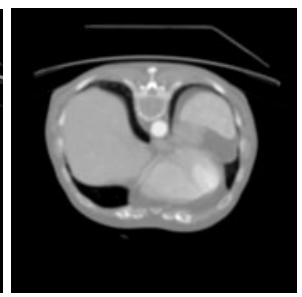

(b)

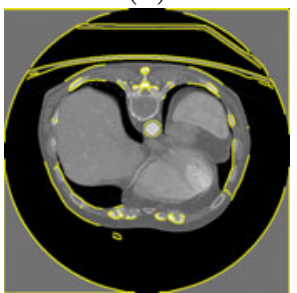

(e)

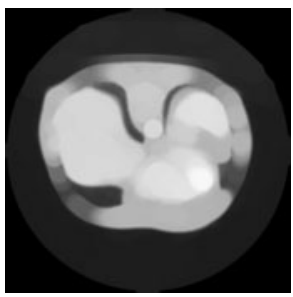

(c)

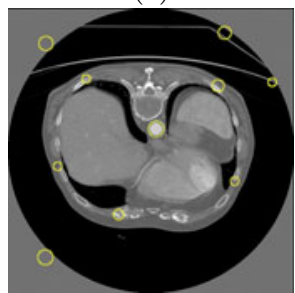

(f)

Fig. 5. Preprocessing steps of a CTA slice: (a) Input 2D cross-section (b) Anisotropic diffusion filtering (c) Opening operator result (d) Result of top-hat operator (e) Canny edges (f) Circle detection by Hough transform.

extracted perpendicularly to the vessel tangent, the vessel boundary resembles a circle. Figure 7 shows the extracted vessel trajectory. For the time, estimated diameter is not employed for visualization, although is immediately available after HT computation. Scale tests were performed after HT determination and vessel radius has been found to be off by +2 or -2 voxels. 

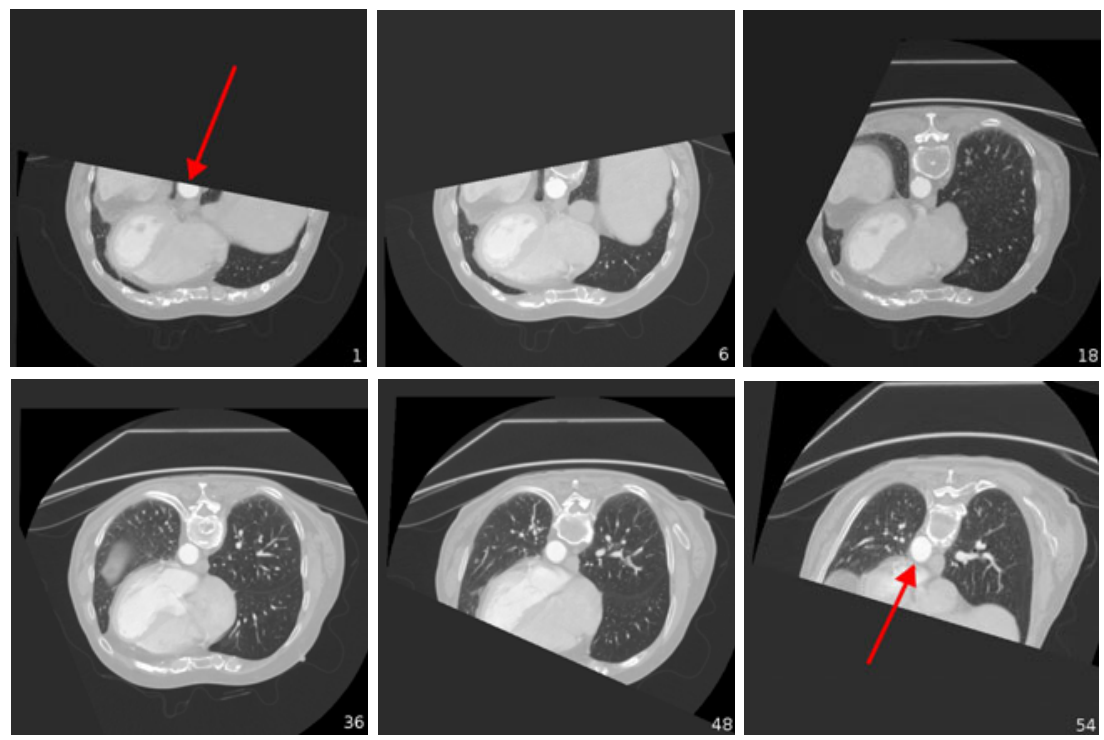

Fig. 6. From left to right, it is shown part of the sequence of $2 \mathrm{D}$ cross-sections extracted during the tracking of the thoracic aorta. The red arrow indicates the aorta cross-section.
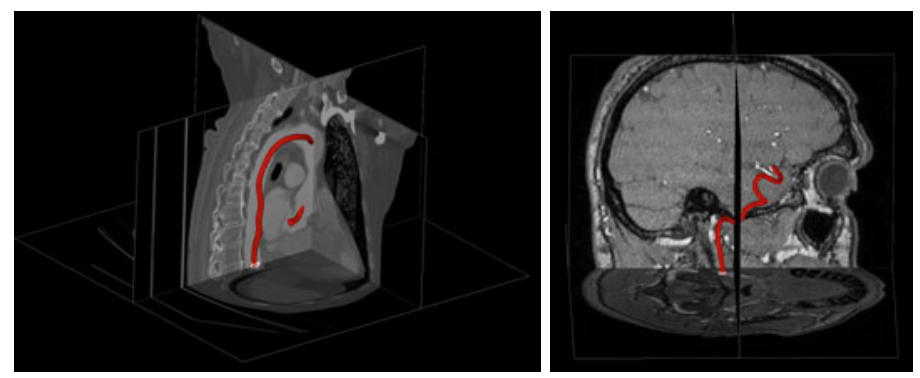

Fig. 7. (a) Thoracic aorta trajectory in CTA image track after 405 successive iterations (b) Carotid artery trajectory in MRA image after 310 iterations. Each centerline was extracted and represented as a cylinder. The step size between centerlines is $\delta=1$ voxel.

\section{Discussion and Conclusions}

In the present study, we have proposed a semi-automated method to detect and track vessel centerlines in CTA and MRA images. The initialization step is currently done interactively in order to select the starting plane for extraction. This interaction can be minimized by choosing a single point inside the vessel or selecting the most perpendicular plane to this vessel.

The methodology has shown to work well under significant amounts of noise, although a branch segment was not identified in the synthetic example corrupted 
with $15 \%$ Gaussian noise. A change in the tracking heuristics will be made in order to account for detecting an inverse direction from the seed point.

The main contribution of this work is the use of HT to define the seed point for tracking and detecting the vessel scale without resorting to multiscale analysis techniques, and in spite of the presence of anomalies, a mean diameter can be established. While branching in the synthetic datasets was handled according to the Section 2, it still remains to be thoroughly validated, given the complexity of real data bifurcations. Bifurcations were not handled in the real datasets.

Future work will focus on fine tuning the scale detection resulting from the HT, reconstructing the diameter of the extracted vessels, automatic bifurcation handling using analysis of curvature of the vessel profile at the cross-section when it approaches the branching point and use of ground-truth data to allow our method to be compared with established methods.

\section{References}

1. American Heart Association: Heart disease and stroke statistics - 2010 update. American Heart Association - Learn and Live (2010)

2. Zhuang, Z.W., Gao, L., Murakami, M., Pearlman, J.D., Sackett, T.J., Simons, M., Muinck, E.D.: Arteriogenesis: Non-invasive quantification with multi-detector row ct angiography and three-dimensional volume rendering in rodents. Radiology 240, 698-707 (2006)

3. Bankman, I., Hopkins, J.: Handbook of Medical Imaging. Elsevier, Amsterdam (2000)

4. Qian, X., Brennan, M.P., Dione, D.P., Dobrucki, W.L., Jackowski, M.P., Breuer, C.K., Sinusas, A.J., Papademetris, X.: A non-parametric vessel detection method for complex vascular structures. Medical Image Analysis 13, 49-61 (2009)

5. Metz, C.T., Weustink, A.C., Mollet, N.R., van Walsum, T., Niessen, W.J.: Coronary centerline extraction from ct coronary angiography images using a minimum cost path approach. Medical Physics 36(12) (2009)

6. Wink, O., Niessen, W.J., Viergever, M.A.: Multiscale vessel tracking. IEEE Transaction on Medical Imaging 23(1) (2004)

7. McIntosh, F., Hamarneh, G.: Vessel crawlers: 3d physically-based deformable organisms for vasculature segmentation and analysis. In: IEEE Conference on Computer Vision and Pattern Recognition, pp. 1084-1091 (2006)

8. Lesage, D., Angelini, E.D., Bloch, I., Funka-Lea, G.: A review of 3d vessel lumen segmentation techniques: Models, features and extraction scheme. Medical Image Analysis (2009)

9. Kirbas, C., Quek, F.: A review of vessel extraction techniques and algorithms. ACM Computing Surveys 36(2), 81-121 (2004)

10. Frangi, A.F., Niessen, W.J., Vincken, K.L., Viergever, M.A.: Multiscale vessel enhancement filtering. In: Wells, W.M., Colchester, A.C.F., Delp, S.L. (eds.) MICCAI 1998. LNCS, vol. 1496, pp. 130-137. Springer, Heidelberg (1998)

11. Manniesing, R., Viergever, A., Niessen, W.J.: Vessel enhancing diffusion. a scale space representation of vessel structures. Medical Image Analysis 10, 815-825 (2006)

12. Alyward, S., Bullitt, E.: Initialization, noise, singularities, and scale in height ridge traversal for tubular object centerline extraction. IEEE Transaction on Medical Imaging 21, 61-75 (2002) 